\title{
Structural Ensemble of the Insulin Monomer
}

\author{
Luis Busto-Moner, Chi-Jui Feng, Adam Antoszewski, Andrei Tokmakoff,* and Aaron R. Dinner* \\ Cite This: Biochemistry 2021, 60, 3125-3136 \\ Read Online
}

ABSTRACT: Experimental evidence suggests that monomeric insulin exhibits significant conformational heterogeneity, and modifications of apparently disordered regions affect both biological activity and the longevity of pharmaceutical formulations, presumably through receptor binding and fibrillation/degradation, respectively. However, a microscopic understanding of conformational heterogeneity has been lacking. Here, we integrate all-atom molecular dynamics simulations with an analysis pipeline to investigate the structural ensemble of human insulin monomers. We find that $60 \%$ of the structures present at least one of the following elements of disorder: melting of the A-chain N-terminal helix, detachment of the B-chain N-terminus, and detachment of the B-chain C-terminus. We also observe partial melting and extension of the B-chain helix and significant conformational heterogeneity in the region containing the B-chain $\beta$-turn. We then estimate hydrogen-exchange protection factors for the sampled ensemble and find them in line with experimental results for KP-insulin, although the simulations underestimate the importance of unfolded states. Our results help explain the ready exchange of specific amide sites that appear to be protected in crystal structures. Finally, we discuss the implications for insulin function and stability.

\section{INTRODUCTION}

The protein hormone insulin regulates glucose uptake in cells, and a lack of its activity due to insufficiency or insensitivity leads to diabetes mellitus, a widespread life-threatening metabolic disease; administration of insulin and its analogues is a key treatment. ${ }^{1,2}$ Insulin is predominantly hexameric and dimeric during production, delivery, and circulation, but it binds to its receptor as a monomer, ${ }^{3}$ in a conformation that differs significantly from that in solution NMR and X-ray crystallographic structures. ${ }^{4,5}$ Conditions and sequence changes that favor the monomeric form promote fibrillation ${ }^{6-9}$ and degradation, ${ }^{10}$ which limit the stability of therapeutic preparations. An understanding of the structures accessible to the monomer under physiological conditions is therefore of great interest in medicine, biophysics, and structural biology.

Available structures for the insulin monomer (e.g., Figure 1, from NMR in water/acetonitrile ${ }^{11}$ ) are consistent with the $\mathrm{T}$ state structure observed in crystallographic studies of the hexamer. ${ }^{12}$ In the monomer, there are 51 residues distributed between two chains, denoted A (21 residues) and B (30 residues). Two disulfide bridges ( $\mathrm{Cys}^{\mathrm{A} 7}-\mathrm{Cys}^{\mathrm{B} 7}$ and $\mathrm{Cys}^{\mathrm{A} 20}$ $\mathrm{Cys}^{\mathrm{B} 19}$ ) connect the two chains, and a third $\left(\mathrm{Cys}^{\mathrm{A}}{ }^{\mathrm{A}}-\mathrm{Cys}^{\mathrm{A} 11}\right)$ is internal to the A chain. The secondary structure of the $\mathrm{T}$ state consists of two $\alpha$-helices in the A-chain, one at the $\mathrm{N}$-terminus $\left(\mathrm{Gly}^{\mathrm{A}}{ }^{-}-\mathrm{Ser}^{\mathrm{A} 9}\right)$ and one at the C-terminus $\left(\mathrm{Leu}^{\mathrm{A13}}-\mathrm{Cys}^{\mathrm{A} 20}\right)$; we term these the AN- and AC-helices, respectively. The B-chain includes one $\alpha$-helix $\left(\mathrm{Gly}^{\mathrm{B} 9}-\mathrm{Cys}^{\mathrm{B} 19}\right)$, which we term the B-helix, and a $\beta$-turn $\left(\mathrm{Gly}^{\mathrm{B} 20}-\mathrm{Gly}^{\mathrm{B} 23}\right)$.

Measurements of amide hydrogen exchange for KPinsulin, ${ }^{13,14}$ an analogue that is monomeric in solution, show little protection at the $\mathrm{A}$-chain $\mathrm{N}$-terminus $(\mathrm{A} 1-\mathrm{A} 9)$, the $\mathrm{B}$ - chain N-terminus ( $\mathrm{Phe}^{\mathrm{B} 1}-\mathrm{Cys}^{\mathrm{B} 7}$ ), and the B-chain $\mathrm{C}$-terminus $\left(\mathrm{Gly}^{\mathrm{B} 20}-\mathrm{Ala}^{\mathrm{B} 30}\right){ }^{15}$ The exchange at these sites indicates that these segments sample solvent-exposed states, though the nature of these states remains unclear. Experimental characterization of the wild-type monomer structural ensemble itself is hindered by both fibrillation and oligomerization at concentrations ranging from micromolar to millimolar. ${ }^{16}$ Experimental structural information about the monomer thus mainly comes from studies of sequences with substitutions, as above, or from studies in solution conditions that differ significantly from physiological ones (e.g., $\mathrm{pH}<3$ and/or with cosolvents), ${ }^{7,11,13,14}$ which can impact the degree of disorder. ${ }^{17}$

Setting aside the issues above, structural characterization of a protein with disordered regions is experimentally challenging because conformations can interconvert on pico- to millisecond lifetimes. ${ }^{18-20}$ Although NMR relaxation measurements can access these timescales to give information about contributing motions, extracting the structures and their populations from chemical shifts is not straightforward due to averaging. ${ }^{21}$ Measurements of Förster resonance energy transfer between dye labels can access such timescales for single molecules, ${ }^{22-24}$ but they are impractical for small proteins with sizes smaller than typical transfer radii, on the scale of the labels themselves.

Received: August 30, 2021

Revised: September 21, 2021

Published: October 12, 2021 

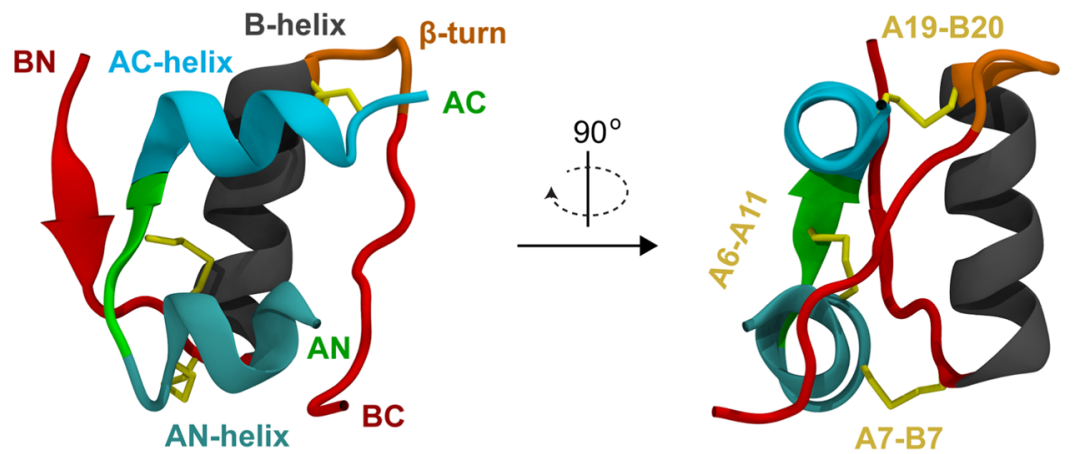

Figure 1. Schematic view of the insulin structure (PDB ID 2JV1 ${ }^{11}$ ). A-chain $\mathrm{N}$ - and C-termini are labeled in green (AN and AC), and B-chain N-and $\mathrm{C}$-termini are labeled in red (BN and BC). The A-chain $\alpha$-helices $\left(\mathrm{AN}, \mathrm{Gly}^{\mathrm{A} 1}-\mathrm{Ser}^{\mathrm{A} 9}\right.$; AC, $\mathrm{Leu}^{\mathrm{A} 13}-\mathrm{Cys}^{\mathrm{A} 20}$ ) are colored teal and cyan, respectively. The Bhelix $\left(\mathrm{Gly}^{\mathrm{B} 9}-\mathrm{Cys}^{\mathrm{B} 19}\right)$ is shown in black. The $\mathrm{Gly}^{\mathrm{B} 20}-\mathrm{Gly}^{\mathrm{B} 23} \beta$-turn is shown in orange. Disulfide bridges are shown in yellow.

\section{Check physical interpretation of $\mathrm{tlCs}$}

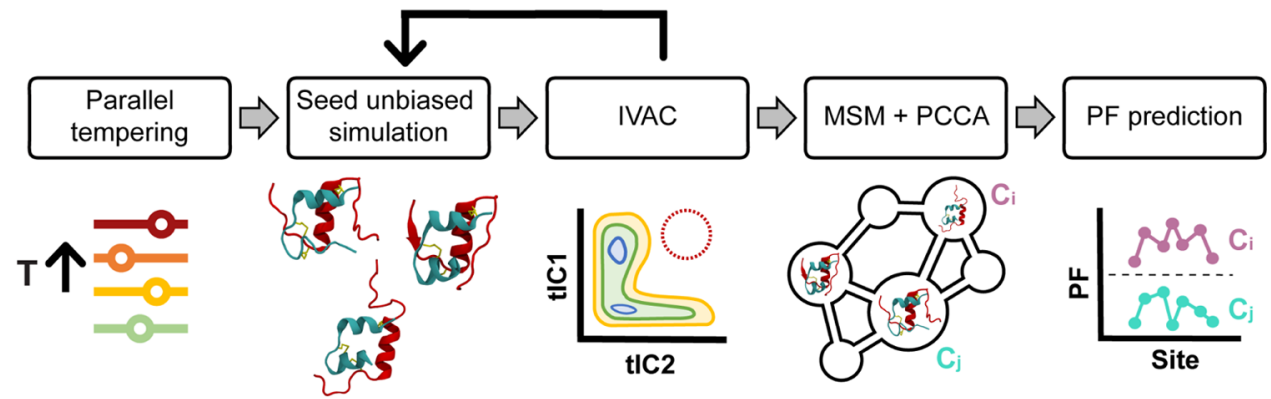

Figure 2. Overview of the pipeline used to sample and cluster structures. The resulting clusters were used to model the protection factors of individual $\mathrm{H}_{\mathrm{N}}$ sites.

Simulations can provide direct access to microscopic structures and the forces stabilizing them, but there have been surprisingly few all-atom molecular dynamics studies of the insulin monomer. Nanosecond-scale unbiased simulations of the porcine insulin monomer starting from the $\mathrm{T}$ state in the hexamer crystal structure ${ }^{12}$ showed disorder in the $\mathrm{N}$ - and $\mathrm{C}$ termini of the B-chain ${ }^{25}$ but not the AN-helix. The root-meansquare deviation from the $\mathrm{T}$ state suggests significant unfolding also in ref 26 , which takes a similar approach, though few structural details are given. Bias-exchange metadynamics simulations of porcine insulin at low $\mathrm{pH}$ and high temperature produced a diverse ensemble of essentially fully unfolded states. $^{27}$

Here, we report a study of the structural ensemble of human wild-type insulin at low $\mathrm{pH}$ and room temperature. We combine enhanced sampling, ${ }^{28,29}$ extensive unbiased simulation, and a variety of analysis methods ${ }^{30-34}$ to ensure a good exploration of the accessible conformational space (Figure 2). The simulations indicate that $60 \%$ of the population under these conditions contains at least one of the following elements: melting of the AN-helix, detachment of the B-chain N-terminus, and detachment of the B-chain C-terminus. We further characterize the Bchain conformations with regard to the structure of the B-helix and the $\beta$-turn, as well as the timescales for interconversion between states, which are on the order of microseconds. Our results are consistent with the hydrogen-deuterium exchange results for KP-insulin described above, ${ }^{15}$ and we use simulated protection factors ( $\mathrm{PFs}$ ) to further assess the contributions from different states. Finally, we discuss how our atomic-resolution description of conformation heterogeneity within the insulin monomer structural ensemble provides insights into insulinreceptor binding and fibrillation.

\section{MATERIALS AND METHODS}

The main goal of this work was to explore the conformational space of the insulin monomer. The pipeline we followed is summarized in Figure 2. A first set of structures was generated using parallel tempering, ${ }^{28,29}$ which was used to seed equilibrium unbiased simulations. The slowest relaxing motions of the protein were then identified using the integrated variational approach for conformational dynamics (IVAC), ${ }^{32,33}$ and low probability structures in that space were subsequently sampled as starting points of new unbiased simulations. The resulting data set was then clustered into 1000 states to build a Markov state model (MSM), ${ }^{30,31}$ which was further coarse-grained using Perron cluster analysis (PCCA). ${ }^{34}$ The resulting 10 clusters characterize the insulin monomer structural ensemble. We used these clusters to estimate the individual $\mathrm{H}_{\mathrm{N}}$ protection factors for comparison with experimental hydrogen-exchange data. ${ }^{15}$

System Setup and Equilibration. The system was modeled with the CHARMM36m force field. ${ }^{35-37}$ All simulations were performed using GROMACS 5.1.4, ${ }^{38}$ and the system was prepared using CHARMM-GUI 2.1; ${ }^{39,40}$ All molecular visualizations were done in VMD. ${ }^{41}$ Unless otherwise noted, simulations were carried out in the isochoric isothermal (NVT) ensemble at $303.15 \mathrm{~K}$ using a Langevin thermostat ${ }^{42}$ with a 2 -fs time step and a friction constant of $10 \mathrm{ps}^{-1}$ applied to all atoms. All bonds to hydrogen atoms were constrained using the LINCS algorithm. ${ }^{43}$ Periodic boundary conditions were employed, and the particle-mesh Ewald method ${ }^{44}$ was used to calculate electrostatic forces with a cutoff distance of $1.2 \mathrm{~nm}$. The Lennard-Jones interactions were smoothly switched off from 1.0 to $1.2 \mathrm{~nm}$ through the built-in GROMACS force-switch function. 
The starting point for our simulations was an NMR structure of human insulin in water/acetonitrile solution (PDB ID 2JV1). ${ }^{11}$ Hydrogens were added to the PDB structure, and it was solvated in a $(7 \mathrm{~nm})^{3}$ box using TIP3P water. ${ }^{45}$ PROPKA $^{46,47}$ was used to estimate the protonation state of individual residues at $\mathrm{pH} 2.5$, within the $\mathrm{pH}$ range commonly used to better solubilize insulin for NMR and IR experiments. Given the resulting charge states, $4 \mathrm{Cl}^{-}$ions were added to neutralize the system. There were 25006 atoms in total.

The system was energetically minimized using the steepest descent method until the maximum force felt by the system was below $1000 \mathrm{~kJ} / \mathrm{mol} \mathrm{nm}$. The system was then equilibrated for $100 \mathrm{ps}$ in the NVT ensemble with a 1 -fs time step, followed by 10 ns in the NPT ensemble at 1 bar using the Parrinello-Rahman barostat, ${ }^{48}$ with a 2 -fs time step and time constant of 5.0 ps. For the energy minimization and equilibration above, harmonic restraints were used to stabilize the positions of all nonhydrogen protein atoms. The system was equilibrated further for $1 \mathrm{~ns}$ in the NPT ensemble without position restraints. We then imposed a box size of $(6.5 \mathrm{~nm})^{3}$, based on the average size of the highest temperature simulations in the parallel tempering (see below). This box size was used for all further simulations, including the unbiased ones. The system was equilibrated once more without position restraints for $1 \mathrm{~ns}$ in the NVT ensemble.

Parallel Tempering. In the absence of a priori knowledge of structural descriptors that could resolve different stable states, we chose to use parallel tempering to enhance conformational exploration. $^{28,29}$ The goal of these simulations was to generate a diverse set of conformations to further seed independent room temperature simulations. A first set of 20 replicas ranging from 303 to $360 \mathrm{~K}$ was used, with temperatures spaced to achieve an exchange probability of $5 \%$ based on the number of atoms. ${ }^{49}$ Before production runs, each simulation was individually equilibrated at the desired temperature following the procedure detailed above. As exchanging replicas must have the same box size, before the final NVT equilibration, we set the box size to the average for the highest temperature simulations $(6.5 \mathrm{~nm})^{3}$. The total length of each individual simulation was $100 \mathrm{~ns}$, with exchanges attempted every 1 ps. Briefly, 19\% of exchanges were accepted. A second set of 20 replicas between 350 and $415 \mathrm{~K}$ was run, with individual simulations lasting $200 \mathrm{~ns}$ and the same exchange attempt rate. Typically, $23 \%$ of exchanges were accepted. From these data, we extracted 15 structures by hand that were representative of the elements of disorder observed and used them to seed free simulations at $303.15 \mathrm{~K}$ for $2.8 \mu \mathrm{s}$.

IVAC. To characterize and visualize the structures, we projected them onto the slowest decorrelating modes of the system identified by a modified version of the variational approach to conformational analysis (VAC). ${ }^{32}$ The resulting time-lagged independent components (tICs) are linear combinations of the input features. Common feature choices are atom positions, distances between selected atoms, and (pseudo)dihedral angles. Here, we used pairwise distances between the $\alpha$-carbons of $\mathrm{Gly}^{\mathrm{A} 1}, \mathrm{Cys}^{\mathrm{A} 6}, \mathrm{Tyr}^{\mathrm{A} 19}, \mathrm{Val}^{\mathrm{B} 2}, \mathrm{Ser}^{\mathrm{B} 9}$, $\mathrm{Tyr}^{\mathrm{B} 16}$, $\mathrm{Gly}^{\mathrm{B} 20}$, $\mathrm{Phe}^{\mathrm{B} 24}$, and $\mathrm{Ala}^{\mathrm{B} 30}$. The idea was to cover the whole protein and all of the observed conformational changes with a reduced initial number of dimensions (36).

In VAC, a lag time needs to be chosen to compute the timecorrelation matrix between input dimensions. The lag time should be long enough for fast processes to decorrelate but short enough to resolve the dynamics of interest. In practice, choosing this lag time can be difficult, and the results can be sensitive to it. We overcome this issue in the present study using integrated
VAC (IVAC), which sums the time-correlation matrices over a range of lag times for greater robustness. ${ }^{33}$

We iteratively applied IVAC with lag times ranging from 1 to $100 \mathrm{~ns}$ to develop a data set with good coverage of the accessible space. First, we applied it to the 15 unbiased $2.8-\mu$ s trajectories from the parallel-tempering seeds; we projected our sampling onto the resulting first three tICs and selected 14 structures from poorly sampled regions to seed additional $2.8-\mu \mathrm{s}$ simulations. We repeated this process (IVAC on the cumulative data set to determine tICS, projection, selection of seed structures, and unbiased simulation), selecting 14 and 16 seed structures; we stopped sampling when the physical meaning of the first 3 tICs (see Results) stabilized. The final data set was thus composed of 59 trajectories, each of $2.8 \mu \mathrm{s}$. This constitutes an aggregate sampling of $0.16 \mathrm{~ms}$. IVAC was run again on the entire data set of $\sim 16$ million time points as the starting point for further analysis.

Clustering, MSM, and PCCA. Each IIC corresponds to a slowly decorrelating mode, and its associated eigenvalue corresponds to a timescale. We observed a spectral gap after the first six tICs (Figure S12a) and thus used those coordinates as the basis for the construction of a Markov state model (MSM). ${ }^{30,31}$ Specifically, we clustered the structures into 1000 microstates using the $k$-means algorithm in PyEMMA 2.5.7. ${ }^{50}$ Using a lag time of $100 \mathrm{~ns}$, we computed the transition matrix between the microstates. The top eigenvectors of the transition matrix were then used to further group the microstates into 10 clusters, using Perron cluster analysis (PCCA). ${ }^{34}$ The MSM was validated as shown in Figure S12b,c.

To test the robustness of the analysis, we projected the centers of the obtained clusters on other IVAC spaces, using different lag-time ranges $(1-20,1-200$, and $1-500 \mathrm{~ns})$ and input basis sets (using 12, 15, and all $\alpha$-carbons excluding neighbors closer than 4 residues). We found that the first $3 \mathrm{tICs}$ separate the main 5 clusters in a consistent manner: $\mathrm{IIC} 1$ separates clusters 0 and 1 , tIC2 separates clusters 0 and 3 , and tIC3 separates clusters 2 and 4. Use of a coarser discretization for the MSM construction ( 500 microstates) and longer MSM lag times (200 ns) yielded similar clusters.

Estimating Protection Factors from the Structural Ensemble. We estimate protection factors for each $\mathrm{H}_{\mathrm{N}}$ site $i$ $\left(f_{i}\right)$ based on the number of hydrogen bonds that each backbone amide forms with an acceptor atom in the protein chain $\left(\mathrm{HB}_{i}^{\mathrm{p}}\right)$ and the number of hydrogen bonds it forms with any water oxygen $\left(\mathrm{HB}_{i}^{\mathrm{w}}\right)$. We use four different models that combine these two quantities in different manners to ensure our results are not specific to a particular model. We refer to the models as Park, Ratio, Difference, and Bound-Difference. They all rely on the idea that an amide site is more protected the more hydrogen bonds it forms with other protein residues and the fewer with water.

The Park model uses the definition proposed by Park et al. ${ }^{51}$

$$
f_{i}=\frac{b}{1+\sqrt{b}\left(\frac{1}{\sqrt{b}}\right)^{\mathrm{NH}_{i}}}=\frac{b}{1+b^{-\left(\mathrm{NH}_{i}-1\right) / 2}}
$$

where $\mathrm{NH}_{i}$ is defined as

$$
\mathrm{NH}_{i}=\min \left(\mathrm{HB}_{i}^{\mathrm{p}}, 1\right)-\min \left(\mathrm{HB}_{i}^{\mathrm{w}}, 1\right)
$$

The base parameter $b$ is an arbitrary constant. Park et al. use values between $10^{4}$ and $10^{6}$, but we set it to 500 to make the maximum $\sim 250$, close to the maximum experimental values in ref 15 .

The Ratio model is 


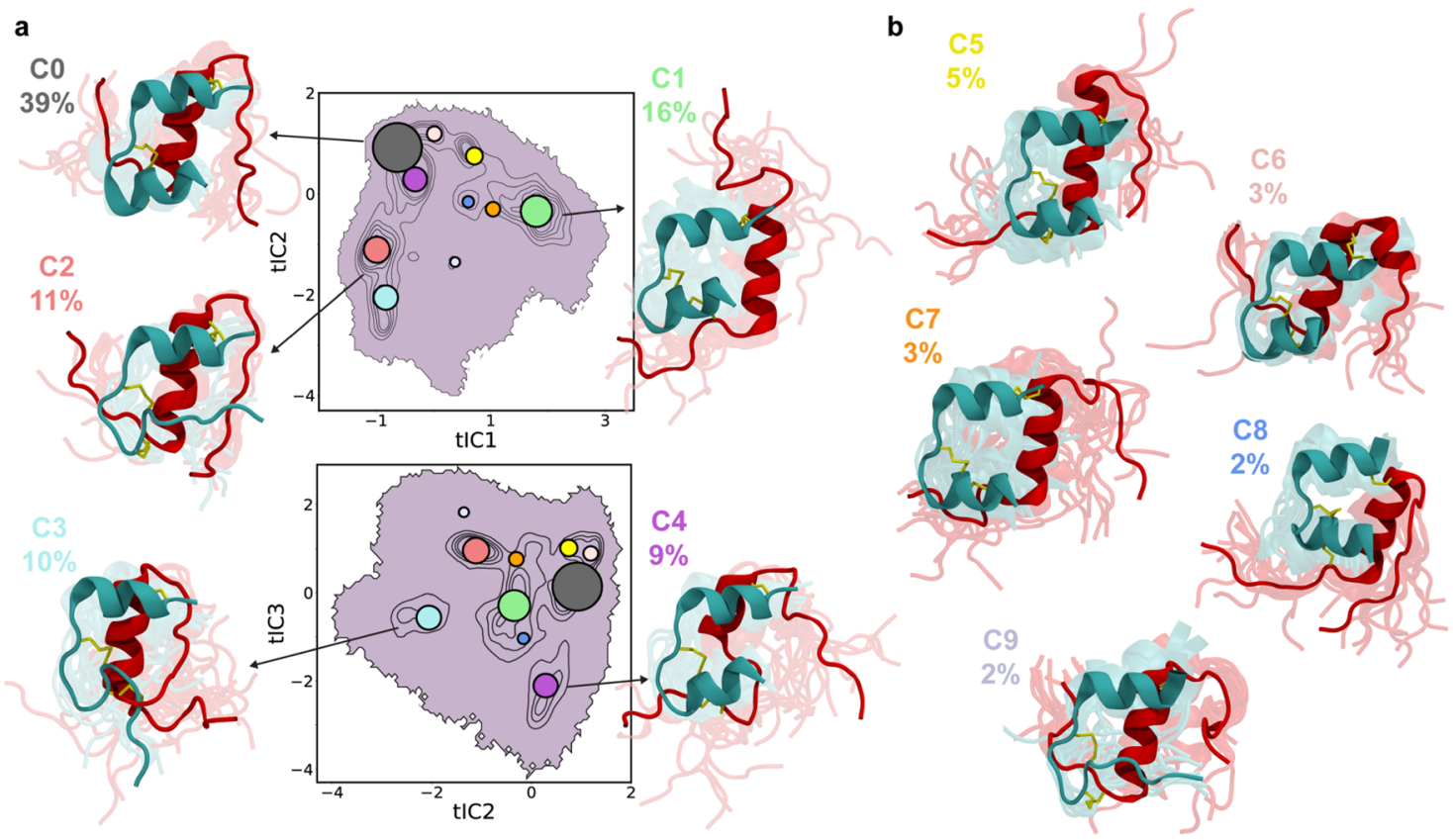

Figure 3. (a) Projection of all sampled structures onto tIC1 vs tIC2 (top) and tIC2 vs tIC3 (bottom), with clusters indicated. Each circle is centered on the median IIC values for a cluster, and the radius is proportional to the physical weight of the cluster. Representative structures for clusters $0-4$, constituting $\sim 85 \%$ of the population, are shown. The A-chain is teal, and the B-chain is red. (b) Clusters 5-9, constituting the remaining $15 \%$ of the population.

$$
f_{i}=\frac{\mathrm{HB}_{i}^{\mathrm{p}}}{\max \left(\mathrm{HB}_{i}^{\mathrm{w}}, 0.02\right)}
$$

where the max function prevents division by zero for structures with $\mathrm{HB}_{i}^{\mathrm{w}}=0$.

The Difference model is

$$
f_{i}=\mathrm{HB}_{i}^{\mathrm{p}}-\mathrm{HB}_{i}^{\mathrm{w}}
$$

Finally, the Bound-Difference model is

$$
f_{i}=\mathrm{NH}_{i}
$$

where $\mathrm{NH}_{i}$ has the same form as in eq 2 . Note that in this case, $f_{i}$ can only take values of 1,0 , or -1 .

To compute $\mathrm{HB}_{i}^{\mathrm{p}}$ and $\mathrm{HB}_{i}^{\mathrm{w}}$, we tested several donor-acceptor distance cutoffs $(0.35,0.40,0.50$, and $0.60 \mathrm{~nm})$ and hydrogendonor-acceptor angle cutoffs $\left(40,50,70\right.$, and $\left.90^{\circ}\right)$. The mean $\mathrm{PF}$ for each $\mathrm{H}_{\mathrm{N}}$ site $i$ for each cluster $j, \mathrm{PF}_{i j}$, was computed by averaging over 4000 sample structures within it. The ensemble protection factor for one site, $\mathrm{PF}_{i}$, is taken as the weighted average across clusters $0-4$.

In general, our results are consistent across model and parameter space. Individual absolute values of $\mathrm{PF}_{i}$ change depending on both the model and the angle-distance cutoffs used to define the hydrogen bonds, but consistent trends are observed (Figure S9). The correlations with experimental values ranged from 0.59 to 0.82 , as shown in Figure S10. We observe that more restrictive angle and distance cutoffs tend to favor counting protein-protein hydrogen bonds, which results in higher protection factors. The correlations are particularly low when a loose angle cutoff is combined with a very restrictive distance requirement because hydrogen bonds of an $\mathrm{H}_{\mathrm{N}}$ with the side chain of its residue are counted, while those with water are neglected.

\section{RESULTS}

Our goal is to characterize the diversity of structures populated by human insulin under low $\mathrm{pH}$ conditions (i.e., with protonated titratable residues), which are commonly used in NMR experiments. To ensure that we adequately sampled the conformational space available to the system, we employed the pipeline in Figure 2, as detailed in Materials and Methods. In brief, we used 15 structures from $6 \mu$ s of parallel tempering, ${ }^{28,29}$ with temperatures ranging from 303 to $415 \mathrm{~K}$ to seed room temperature $(303.15 \mathrm{~K})$ unbiased molecular dynamics simulations, each of which was $2.8 \mu \mathrm{s}$. Based on the projection of these data onto the first three time-lagged independent components (tICs), ${ }^{32,33}$ we iteratively expanded this data set by seeding additional trajectories until physical interpretations of the first three tICs (discussed below) stopped changing. The final data set had 59 trajectories totaling $165.2 \mu$ s of unbiased simulation, with structures saved every 10 ps for more than 1.6 million structures in total. We projected the structures onto the first six tICs and clustered them into 1000 states using the $k$ means algorithm. We then constructed a Markov state model $^{30,31}$ (MSM) and used the PCCA algorithm ${ }^{34}$ to group the MSM microstates into 10 clusters based on the MSM transition matrix. We label the resulting clusters 0-9 in order of decreasing population. The analysis that we present is based on these 10 clusters, which range in population from 38.7 to $1.5 \%$ (see Table S1) and more specifically on the five major ones with populations greater than $9 \%$, which account for $\sim 85 \%$ of the total population.

Structural Features of the Clusters. Representative structures from the 10 clusters are illustrated in Figure 3. The AC-helix and the Leu ${ }^{\mathrm{B} 15}-\mathrm{Cys}^{\mathrm{B} 19}$ segment of the B-helix are wellfolded in all clusters. Otherwise, the clusters differ, with the conformational heterogeneity taking three main forms: ANhelix melting, detachment of the B1-B7 segment, and detachment of the B20-B30 segment. Each such element is 
Table 1. Summary of the Observed Structural Elements in Clusters 0-9 (C0-C9), Expressed as the Physically Weighted Percentage of Structures Presenting a Given Element of Disorder within Each Cluster ${ }^{a}$

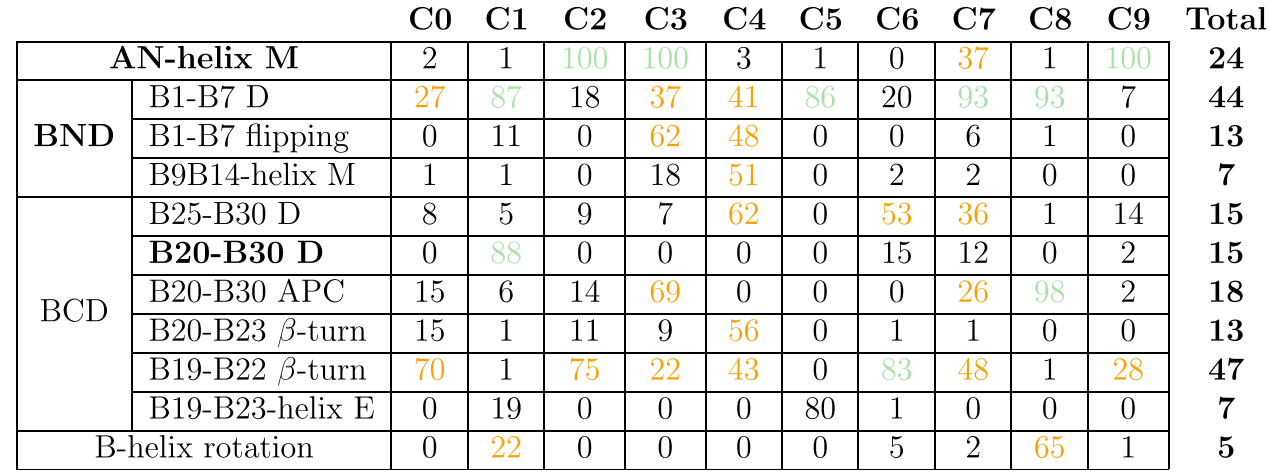

${ }^{a}$ Green indicates that $80 \%$ or more of structures within a cluster contain a given element, while orange indicates $20-80 \%$. Total percentages in the ensemble are listed in the last column. Structural elements that correlate with the first three tICs are shown in bold. Abbreviations: BND, B-chain $\mathrm{N}$-terminus detachment; BCD, B-chain C-terminus detachment; D, detachment; M, melting; E, extension; APC, A-plane crossing.

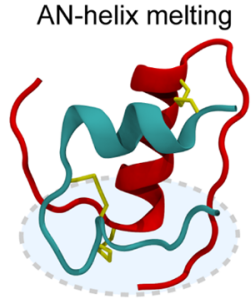

B1-B7 detachment

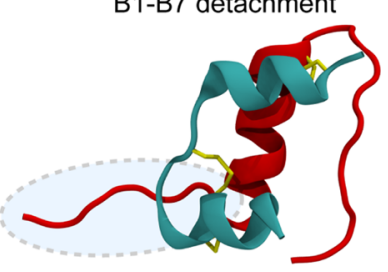

B19-B22 $\beta$-turn

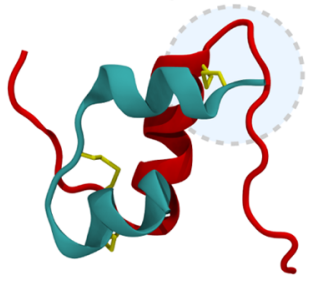

B1-B7 flipping

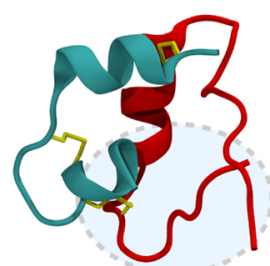

B9B14-helix melting

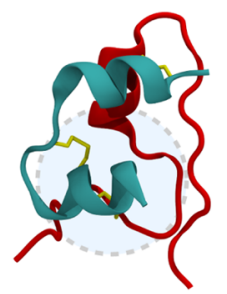

B-helix rotation
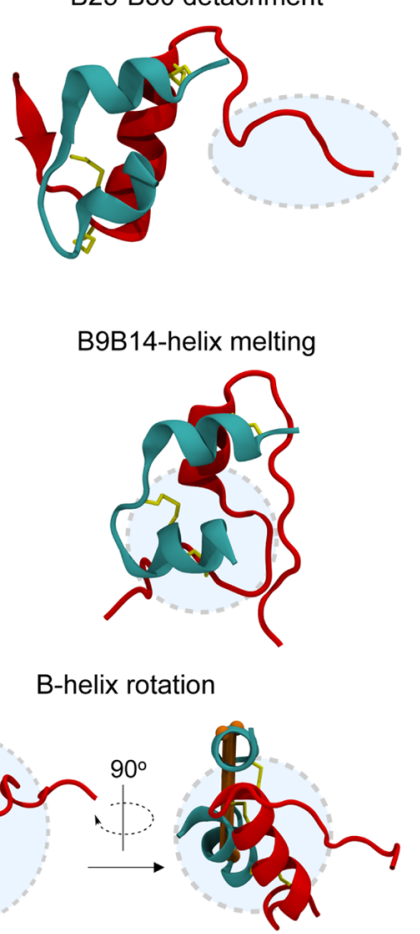

B20-B30 A-plane crossing
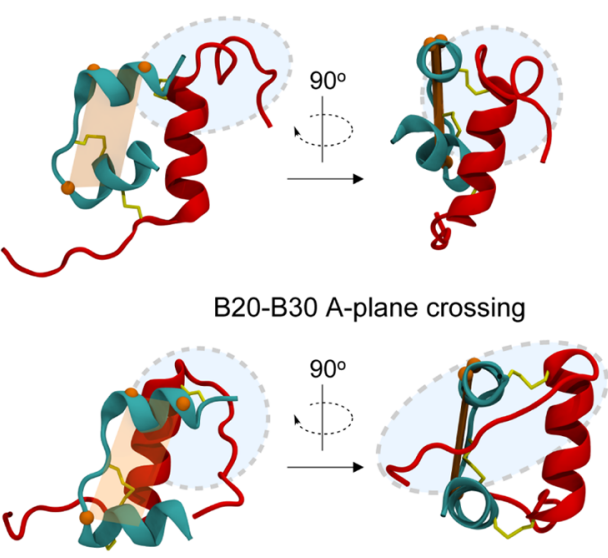

$\checkmark$

.
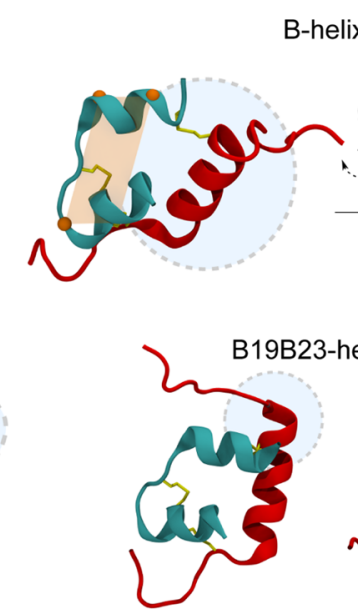

Figure 4. Structures illustrating the elements of disorder that we observe in clusters $0-9$, which are summarized in Table 1. Further details regarding conformational heterogeneity of the B19-B23 segment are shown in Figure S3. B-helix rotation and B20-B30 A-plane crossing are defined with respect to the A-plane, which contains the $\mathrm{C}_{\alpha}$ atoms of $\mathrm{A} 9, \mathrm{~A} 13$, and A20. The three atoms and the A-plane are shown in orange in the relevant panels, as well as in the B20-B30 detachment panel for comparison. Two different structures are shown for B19-B23-helix extension, corresponding to the structures seen in clusters 1 (left) and 5 (right).

generally present or absent in all of the structures in a given cluster. Below, we describe the clusters in more detail, including additional elements that are present in only a fraction of the structures within a cluster. An example is the melting of the
$\mathrm{Ser}^{\mathrm{B} 9}-\mathrm{Ala}^{\mathrm{B} 14}$ region of the B-helix, which is present in only $51 \%$ of structures in cluster 4 . These results are summarized in Table 1 , and example structures are shown in Figures 4 and S3. The collective variables used to determine the presence of the 
elements are described in the Supporting Information and shown in Figure S4.

Cluster 0 represents $38.7 \%$ of the population. It contains structures consistent with the $\mathrm{T}$ state, though some exhibit detachment of B1-B7 (27\%). We find that in most structures of cluster $0(70 \%)$, the $\beta$-turn shifts from $\mathrm{B} 20-\mathrm{B} 23$ (as in the $\mathrm{T}$ state) to $\mathrm{Cys}^{\mathrm{B} 19}-\mathrm{Arg}^{\mathrm{B} 22}$, and the $\mathrm{B} 23 \mathrm{H}_{\mathrm{N}} \cdots \mathrm{O}=\mathrm{C} \mathrm{B} 20$ hydrogen bond is replaced by a $\mathrm{B} 22 \mathrm{H}_{\mathrm{N}} \cdots \mathrm{O}=\mathrm{C} \mathrm{B} 19$ hydrogen bond (Figure S3 Top and Middle). The $\phi$ dihedral angle of Gly ${ }^{\mathrm{B} 23}$ accordingly changes from $\sim 80$ to $\sim-70^{\circ}$ (Figure S4).

Cluster 1 represents $16.0 \%$ of the population and contains structures in which the B-chain residues after B20 are detached, disrupting the B20-B23 $\beta$-turn. The detached B-chain Cterminus can take many conformations and can even align with the AC-helix (Figure 3a). Briefly, 19\% of the structures in cluster 1 exhibit an extension of the B-helix up to $\mathrm{Gly}^{\mathrm{B} 23}$ (B19-B23helix extension). In $87 \%$ of the structures of cluster 1 , the Bchain N-terminus detaches at B7, breaking native inter-chain contacts between the $\mathrm{B} 1-\mathrm{B} 7$ and the $\mathrm{A} 6-\mathrm{A} 11$ segments, as described below. In $22 \%$ of the structures in cluster 1, the B-helix rotates with respect to the A-chain (Figure 4, B-helix rotation). In particular, the top part of the B-helix rotates outward from the plane containing the $C_{\alpha}$ atoms of $A 9, A 13$, and A20, which we call the A-plane. These atoms and the A-plane are shown in orange in the B20-B30 detachment and B-helix rotation panels of Figure 4. An inspection of the trajectories reveals that structures with and without this rotation do not readily interconvert. Clustering the MSM into 12 states instead of 10 splits cluster 1 into two clusters, one with the rotation and one without (1a and 1b, respectively, in Figure S1).

Cluster 2 represents $11.0 \%$ of the population and contains structures in which the AN-helix is melted while the B-chain is mostly native-like. Similar to cluster 0 , the $\beta$-turn predominantly shifts to B19-B22 (75\%).

Cluster 3 represents $9.6 \%$ of the population and combines a melting of the AN-helix (as in cluster 2) with either partial or complete B-chain N-terminus detachment. Partial detachment (37\%) leads to B1-B7 conformations similar to those in cluster 1. Total detachment (62\%) allows $\mathrm{Phe}^{\mathrm{B} 1}-\mathrm{His}^{\mathrm{B} 5}$ to go around the B-helix and form non-native hydrophobic contacts with $\mathrm{Val}^{\mathrm{B} 12}$ and $\mathrm{Tyr}^{\mathrm{B} 16}$. $69 \%$ of the structures in cluster 3 present detachment of the B20-B30 segment toward the AC-helix, i.e., outward from the previously defined A-plane (Figure 4, B20-B30 A-plane crossing). This A-plane crossing (APC) breaks the $\mathrm{B} 20-\mathrm{B} 23 \beta$-turn and changes the $\mathrm{Glu}^{\mathrm{B} 21} \phi$ dihedral angle (Figure S4). A non-native hydrogen bond, $\mathrm{B} 21 \mathrm{H}_{\mathrm{N}} \cdots \mathrm{O}=$ $\mathrm{C}$ B16, is also formed (Figure S3 Bottom). This feature is present in clusters 0 and 2 as well but seems to be facilitated by the combined melting of the AN-helix and the flip of the B1-B7 segment (Figure S6).

Cluster 4 represents $9.4 \%$ of the population and contains structures in which the N-terminus of the B-chain is partially $(48 \%)$ or wholly $(51 \%)$ detached. It also presents considerable disorder in the $\mathrm{Phe}^{\mathrm{B} 25}-\mathrm{Ala}^{\mathrm{B} 30}$ segment $(62 \%)$, and among the major clusters is the one where the B20-B23 $\beta$-turn is more prevalent $(56 \%)$. In approximately half of cluster 4 , the B-helix is melted between $\mathrm{Gly}^{\mathrm{B} 9}$ and $\mathrm{Ala}^{\mathrm{B} 14}$; such structures are rarely found in other clusters, except a small fraction of those in cluster 3. Nevertheless, they are consistent with the observation that serine substitution at B8 impacts not only B1-B7 but also NOEs among B9-B14. ${ }^{52}$

We summarize the clusters with populations below 5\% (Figure 3 b) as follows. Cluster 5 (4.7\%) contains structures in which the B-helix extends to B23, but, unlike cluster 1, with the rest of the B-chain C-terminus packed against the B-helix (see Figure 4 B19-B23-helix extension, right). Cluster 6 (3.5\%) contains structures with $\mathrm{Arg}^{\mathrm{B} 22}-\mathrm{Thr}^{\mathrm{B} 27}$ folded into a separate $\alpha$ helix that packs against the B-helix; in other structures, the Bhelix extends to B25. In structures in cluster 7 (3.4\%), the Bchain $\mathrm{N}$-terminus is partially detached, and the B20-B23 $\beta$-turn is disrupted. The remainder of the B-chain C-terminus is mostly attached. Cluster $8(2.2 \%)$ contains structures with a partial detachment of the B-chain $\mathrm{N}$-terminus and rotation of the $\mathrm{B}$ helix across the A-plane. A-plane crossing of the B20-B30 segment is present in $98 \%$ of cluster 8 , facilitated by this same rotation. Finally, cluster $9(1.5 \%)$ combines melting of the ANhelix (as in cluster 2 ) with loss of the B20-B23 $\beta$-turn and partial detachment of the B-chain C-terminus.

Physical Interpretation of tICs. Since tICs characterize the slowest collective motions in the system, we expect the leading tICs to distinguish the conformational changes separating major clusters. Consistent with this idea, we see that $\mathrm{IIC1}$ separates cluster 0 (native-like) from cluster 1 (B20-B30 detachment); tIC2 separates cluster 0 from cluster 2 (melting of the AN-helix) and cluster 3 (melting of the AN-helix and detachment of the B1-B7 segment); tIC3 separates cluster 2 from cluster 4 (B1B7 detachment). Based on these, we tentatively associated tIC1 with detachment of the B-chain C-terminus, tIC2 with melting of the AN-helix, and tIC3 with detachment of the B-chain Nterminus around B7 (see Figure S1 for tIC4). To confirm these relationships, we characterized the distances between the $\mathrm{C}_{\alpha}$ atoms, hydrogen bonds, secondary structure content, and contact numbers. Multiple measures were highly correlated (Pearson's $|R| \geq 0.8$ ) with each of the first three tICs (Figure S2), which confirmed our hypotheses.

Kinetics. Both the MSM and the IVAC analysis that produce the tICs yield timescales of relaxation within the population. Although the relative timescales of different modes of relaxation are consistent across methods, we find the absolute timescales differ by up to an order of magnitude and are sensitive to algorithmic choices such as the number of states included. We thus report ranges for the timescales associated with the main elements of disorder: $2.0-20.4 \mu$ s for B20-B30 detachment, $0.9-7.3 \mu \mathrm{s}$ for AN-helix melting, and $0.6-6.0 \mu \mathrm{s}$ for B1-B7 detachment. These timescales are of the same order as ones for hairpin folding $(1-20 \mu \mathrm{s})$ and helix formation $(1-2 \mu \mathrm{s}) .^{53}$

One way of visualizing the relaxation of the population is through a network plot in which the thicknesses of the edges are proportional to the fluxes between clusters at equilibrium (Figure 5). This shows that there are two subnetworks between which exchange is slower (the first timescale range above) and within which exchange is faster (the other two timescale ranges above). One subnetwork is composed of clusters $0,2,3,4$, and 6 , and the other subnetwork is composed of clusters 1,5 , and 7 . The fluxes between clusters $0,2,3$, and 4 indicate that exchange from cluster 0 (native-like) to cluster 3 (with AN-helix melting and B1-B7 detachment) proceeds stepwise. Either the B-chain $\mathrm{N}$-terminus first detaches and then the AN-helix melts $(\mathrm{CO} \rightarrow$ $\mathrm{C} 4 \rightarrow \mathrm{C} 3)$, or the other way around $(\mathrm{C} 0 \rightarrow \mathrm{C} 2 \rightarrow \mathrm{C} 3)$. The dynamics within the subnetwork containing clusters 1,5 , and 7 are less straightforward to interpret but appear to involve rearrangements of the $\mathrm{B} 20-\mathrm{B} 30$ segment, including the $\beta$-turn.

Connection to Hydrogen-Exchange Measurements. To validate the structural ensemble and illustrate its utility in interpreting experimental measurements, we used the structures to estimate hydrogen-deuterium exchange protection factors 


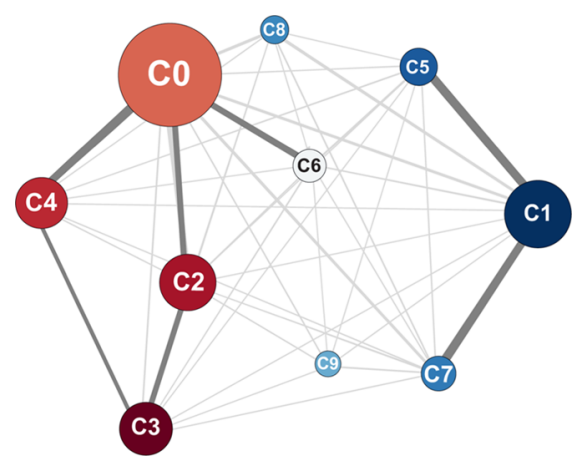

Figure 5. Network plot illustrating population fluxes between clusters 0 and 9. The node radii are proportional to the clusters' equilibrium populations and are positioned using the ForceAtlas algorithm ${ }^{54}$ such that states which exchange rapidly are closer to each other. Edge thicknesses are proportional to the fluxes between nodes and fluxes over $25 \%$ of the maximum flux, which is between clusters 0 and 4 , are highlighted. The nodes are colored according to the cluster mean value of the first nontrivial eigenvector of the MSM transition matrix.

for amide hydrogen $\left(\mathrm{H}_{\mathrm{N}}\right)$ sites. The classical model of exchange assumes that exchange occurs after a site undergoes a transition from a protected (closed) to a solvent-exposed (open) state ${ }^{55,56}$

$$
\mathrm{H}_{\mathrm{N}}^{\text {closed }} \underset{k_{\mathrm{cl}}}{\stackrel{k_{\mathrm{op}}}{\rightleftharpoons}} \mathrm{H}_{\mathrm{N}}^{\text {open }} \stackrel{k_{\text {int }}}{\longrightarrow} \mathrm{D}_{\mathrm{N}}^{\text {open }}
$$

where $k_{\mathrm{op}}$ and $k_{\mathrm{cl}}$ are the opening and closing rates, respectively, and $k_{\text {int }}$ is the intrinsic rate of exchange for a given residue. Using either NMR or mass spectrometry, experiments measure the apparent rate at which protons exchange $\left(k_{\text {obs }}\right)$, which can be expressed as

$$
k_{\mathrm{obs}}=\frac{k_{\mathrm{op}} k_{\mathrm{int}}}{k_{\mathrm{op}}+k_{\mathrm{cl}}+k_{\mathrm{int}}} \simeq \frac{k_{\mathrm{op}} k_{\mathrm{int}}}{k_{\mathrm{op}}+k_{\mathrm{cl}}}=\frac{k_{\mathrm{int}}}{1+\mathrm{PF}}
$$

where the so-called Protection Factor (PF) is defined as PF = $k_{\mathrm{cl}} / k_{\mathrm{op}}$. The approximation made in eq 7 is that $k_{\mathrm{cl}} \gg k_{\text {int }}$, which is known as the EX2 regime. ${ }^{57}$ This is equivalent to saying that the site undergoes many opening and closing events before the exchange, which is consistent with the microsecond-timescale kinetics discussed in the previous section. Since residue intrinsic rates are available from measurements on model peptides, $k_{\mathrm{obs}}$ measurements can be used to obtain $\mathrm{PF}$ for each $\mathrm{H}_{\mathrm{N}}$ site.
Although in principle, the equilibrium between the closed and open states of each site is available from molecular dynamics simulations, in practice, it is not straightforward to define the closed and open states. Qualitatively, they are expected to depend on the site's exposure to the solvent, as well as its hydrogen bonds. ${ }^{57,58}$ Existing models for estimating protection factors incorporate these ideas in ad hoc ways (see refs 51, 59 for reviews and comparisons of models). We tested several such models, as described in Materials and Methods. The results we report in the main text are for the model that yielded the best overall agreement with the available data, but in general, the trends that we observe are consistent across models. We compare the simulated PFs to measurements for KP-insulin, ${ }^{15}$ which is predominantly monomeric in solution.

Overall, the protection factors computed for the ensemble (Figure 6) are in reasonable agreement with the measurements (Pearson's $R=0.82$ ). In both simulation and experiment, the largest PFs occur around A16-A19 and B15-B19. These sites are located in the AC-helix and the part of the B-helix that is wellfolded throughout our simulations (Figure S8). Experimentally, Hua et al. ${ }^{15}$ found the rest of the sites to have relatively low protection factors. In our case, we see a similar trend but overestimate the protection factors of A5-A9, A11, A21, B4, B6, $\mathrm{B} 11, \mathrm{~B} 13, \mathrm{~B} 22$, and B25. Notably, we compute intermediate to high protection factors for A5-A9 within the AN-helix. This is due to cluster 0 , which is native-like and dominates averages due to its large population.

Protection Factors of Individual Clusters. From cluster to cluster, the simulated protection factors of some sites vary significantly (Figure S11). Specifically, sites with variances that are at least 10 times their means are shown in Figure $7 \mathrm{a}$. These sites correspond closely to the largest deviations between the computed protection factors and experimental values. Hua et al. ${ }^{15}$ noted the surprisingly low protection factors of most of these sites (plus B23, which is consistently exposed in our data set) given that they appear to form protein-protein hydrogen bonds in the $\mathrm{T}$ state. They attributed their findings to local conformational fluctuations, such as detachment of the B-chain $\mathrm{N}$-terminus or melting of the AN-helix, which is consistent with the structures that we observe.

In this section, we discuss how the exposure of the sites with highly variable PFs relates to the conformational heterogeneity that we observe. We find that there are five groups of correlated sites, as depicted in Figure 7 in various colors, and we organize

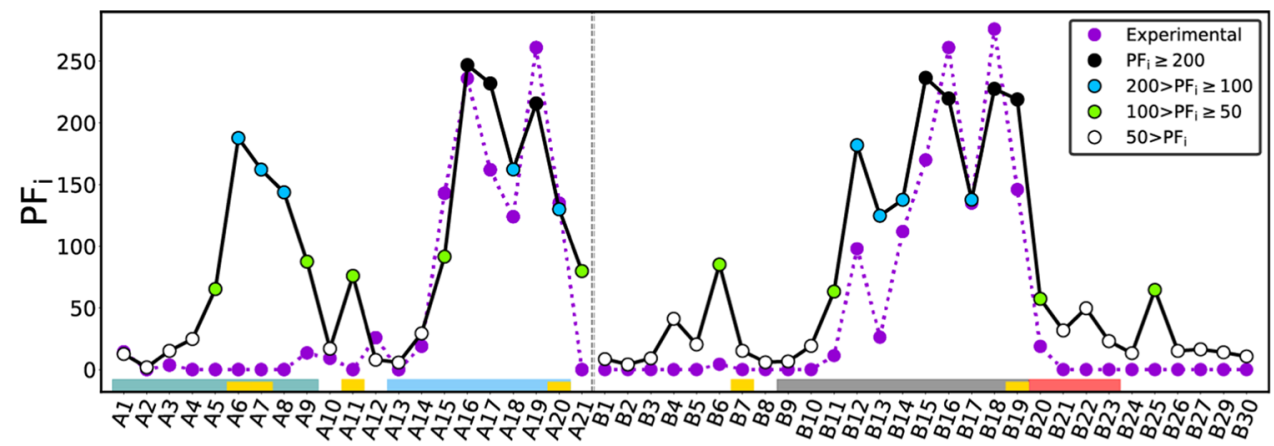

Figure 6. Ensemble protection factor $\mathrm{PF}_{i}$ for each $\mathrm{H}_{\mathrm{N}}$ site $i$, taken as the weighted average for clusters 0 to 4 . Different colors are used depending on the computed PF (see legend). Experimental protection factors come from Hua et al. ${ }^{15}$ Results shown are for the Park model, ${ }^{51}$ with hydrogen-bond hydrogen-donor-acceptor angle and donor-acceptor distance cutoffs of $70^{\circ}$ and $0.5 \mathrm{~nm}$, respectively. Secondary structure motifs of insulin are indicated in the lower part as colored bars, following the color code in Figure 1. 
a

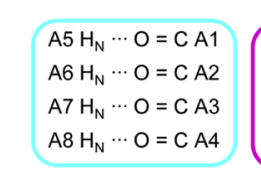

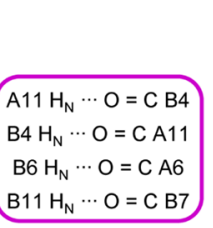

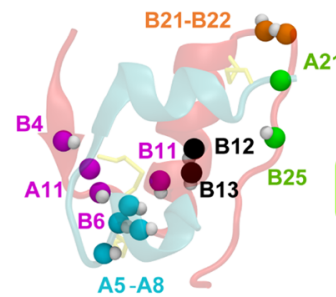

A21

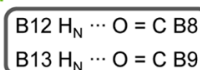

B13 $\mathrm{H}_{\mathrm{N}} \cdots \mathrm{O}=\mathrm{C}$ B9

A21 $\mathrm{H}_{\mathrm{N}} \cdots \mathrm{O}=\mathrm{C}$ B23

$\mathrm{B} 25 \mathrm{H}_{\mathrm{N}} \cdots \mathrm{O}=\mathrm{CA}$ 19

$B 22 \mathrm{H}_{\mathrm{N}} \cdots \mathrm{O}=\mathrm{C}$ B19

b

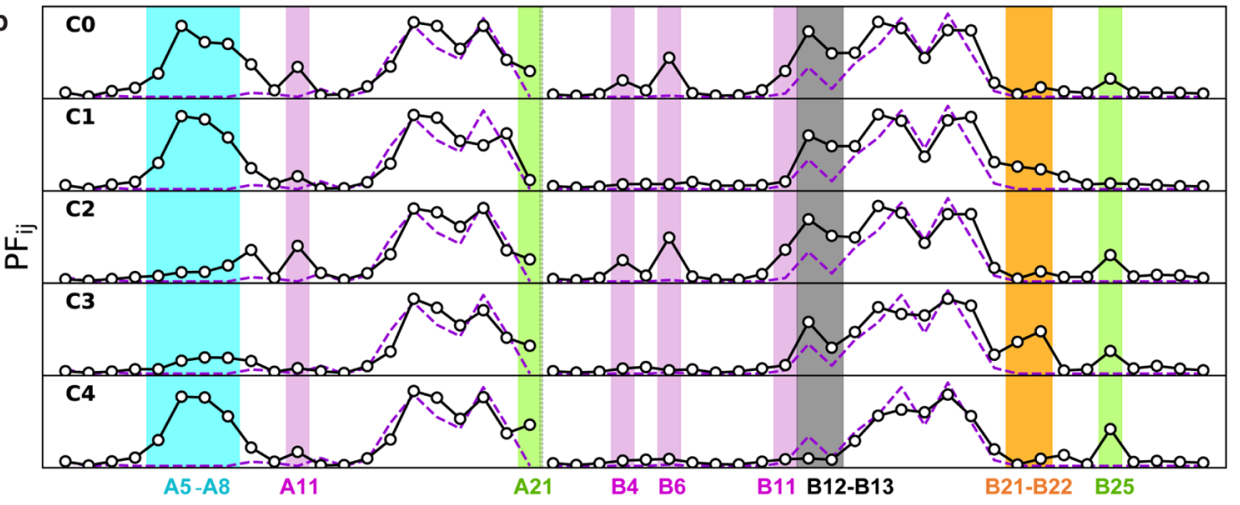

c
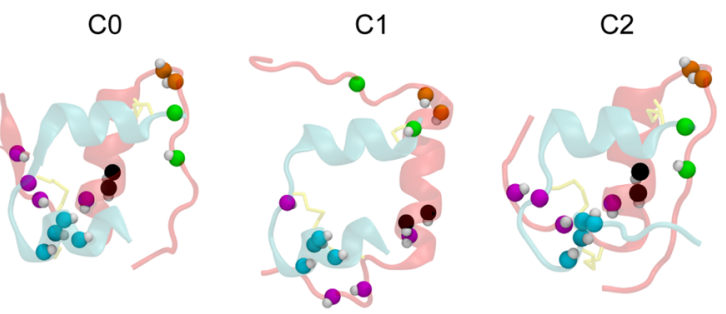

C3

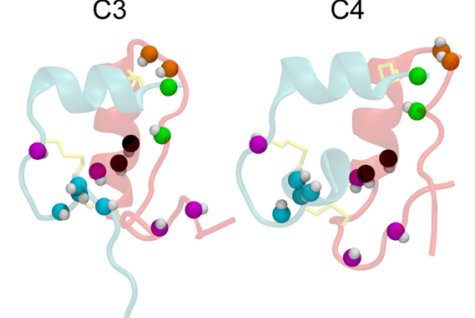

Figure 7. Mean protection factor for individual clusters 0 to 4 and representative structure of each cluster. $\mathrm{H}_{\mathrm{N}}$ sites presenting a coefficient of variation in $\mathrm{PF}_{i}$ of greater than or equal to $10 \%$ are depicted in the structures using various colors for $\mathrm{N}$ atoms and white for $\mathrm{H}$ atoms. (a) Native structure resulting from minimization and solvation of the NMR structure (PDB ID 2JV1 ${ }^{11}$ ). $\mathrm{H}_{\mathrm{N}}$ sites of interest are represented, and their native hydrogenbond state is listed..$^{25}$ (b) Mean simulated protection factor (white circles) for clusters 0 to 4 . Experimental values are plotted as a purple dashed line. Sites of interest are highlighted following the code in panel a. (c) Representative structures for clusters 0 to 4 .

our discussion around them. Results using a different model are shown in Figure S9b.

1. The sites in cyan (A5-A8) are in the AN-helix. Accordingly, they are highly protected in clusters 0,1 , and 4 (each with a largely conserved AN-helix), and exposed in clusters 2 and 3 (each with a predominantly melted AN-helix).

2. The sites in magenta $(\mathrm{A} 11, \mathrm{~B} 4, \mathrm{~B} 6$, and $\mathrm{B} 11)$ are exposed by detachment of the $\mathrm{B}$-chain $\mathrm{N}$-terminus. They are protected in clusters 0 and 2 , which maintain native interchain (e.g., $\mathrm{A} 11 \mathrm{H}_{\mathrm{N}} \cdots \mathrm{O}=\mathrm{C} \mathrm{B} 4$ ) and intra-chain (e.g., $\mathrm{B} 11 \mathrm{H}_{\mathrm{N}} \cdots \mathrm{O}=\mathrm{C}$ B7) hydrogen bonds, but are unprotected in clusters 1,3 , and 4 , which break these hydrogen bonds upon B-chain N-terminus detachment.

3. The sites in black (B12 and B13) are in the B-helix. Protected in almost all of the observed clusters, these sites are exposed by the partial melting of the B-helix, a feature unique to cluster 4 .

4. The sites in green (A21 and B25) are exposed by the detachment of the B20-B30 segment. Moderately protected in clusters $0,2,3$, and 4 , in which B20-B25 remain close to the protein core forming inter-chain hydrogen bonds, they are unprotected in cluster 1 , in which B20-B30 detachment is most prevalent.

5. The sites in orange (B21-B22) are in the $\beta$-turn. These sites are solvent-exposed in clusters 0,2 , and 4 , in which the $\beta$-turn shifts to B19-B22 (Figure S3). These sites are moderately protected in cluster 1 , owing to the extension of the B-helix to B23, and in cluster 3, owing to B20-B30 A-plane crossing, which results in a $\mathrm{B} 21 \mathrm{H}_{\mathrm{N}} \cdots \mathrm{O}=\mathrm{C} \mathrm{B} 16$ hydrogen bond and burial of B22.

\section{DISCUSSION}

Here, we used all-atom molecular dynamics simulations and enhanced sampling methods together with kinetic clustering of conformational substates to provide a microscopic view of insulin's structural ensemble in solution. Our study reveals various forms of conformational variation and a higher degree of disorder than indicated in prior structural studies. The most prominent elements of disorder are AN-helix melting, B-chain $\mathrm{N}$-terminus detachment, and B-chain C-terminus detachment. These elements are associated with microsecond-scale exchange kinetics between the clusters. The B-chain is further characterized by a diversity of conformations at B1-B7, melting of the B-helix at B9-B14, and diversity of conformations at $\mathrm{B} 19-\mathrm{B} 23$, which contains the $\beta$-turn that governs B-chain hinging. Although we model insulin at low $\mathrm{pH}$ because those solution conditions are used experimentally to stabilize the monomer, we expect the disorder that we observe to be present at neutral $\mathrm{pH}$ as well. ${ }^{60}$

We were able to observe the extensive disorder in the structural ensemble owing to the enhanced sampling procedures 
that we used, which combined parallel tempering with selectively seeding relatively long $(2.8 \mu \mathrm{s})$ unbiased simulations based on the tICs for an aggregate sampling of $165 \mu \mathrm{s}$. Our results are consistent with those of Zoete et al., ${ }^{25}$ who performed two unbiased simulations of 5-10 ns of the porcine insulin monomer starting from crystallographic $\mathrm{T}$-state structures. The disorder they reported at the $\mathrm{B} 1-\mathrm{B} 7$ and $\mathrm{B} 25-\mathrm{B} 30$ segments, as well as the shift of the B20-B23 $\beta$-turn to B19-B22, is encompassed in the structures we observe in native-like cluster 0 , consistent with the short duration of their simulations compared with the microsecond timescales we estimate. The conformations in the other nine clusters have not been observed in previous simulation studies.

By simulating protection factors, we show that our results are consistent overall with previous hydrogen-exchange measurements. ${ }^{15}$ Although the ensemble average overestimates the protection of certain $\mathrm{H}_{\mathrm{N}}$ sites, individual clusters provide microscopic structures that are consistent with experimental data. They thus provide support for the hypothesis that conformational heterogeneity in specific regions is the origin of the unexpectedly low protection of these sites. ${ }^{15}$ At the same time, the fact that we overestimate the protection of these sites suggests that we underestimate the extent of disorder in the ensemble. This may reflect issues with the force field (e.g., helix over-stabilization ${ }^{61,62}$ ), sampling, and the model for estimating protection factors from the structures. The specific insulin sequences are also different (wild-type human insulin versus $\mathrm{KP}$ insulin), and it is possible that the sequence substitutions $\left(\right.$ Pro $^{\mathrm{B} 28} \rightarrow \mathrm{Lys}^{\mathrm{B} 29}$ and $\mathrm{Lys}^{\mathrm{B} 29} \rightarrow \mathrm{Pro}^{\mathrm{B} 28}$ ), which are in the B-chain C-terminus, destabilize the nearby AN-helix. That said, our work underscores the importance of sampling key unfolded (open) states for PF computation; indeed, the consistency of our results across different models for estimating PFs from the structures suggests the structural ensemble may have more impact than the choice of model.

Intrinsically disordered regions of proteins are particularly challenging to characterize experimentally, and thus there are limited avenues to compare our ensemble to solution-phase experimental data. ${ }^{63}$ Hydrogen-exchange measurements are made on timescales of minutes to hours, which is orders of magnitude slower than the interconversion between states. Indeed all NMR techniques that employ chemical shift measurements are motionally averaged over the timescale of the measurement, typically $0.1-10 \mathrm{~s}$. One approach that should prove useful for distinguishing rapidly interconverting protein conformations is amide I infrared spectroscopy of specifically labeled backbone carbonyls. The frequency and lineshape of the carbonyl resonance report on hydrogen bonding to the carbonyl and water exposure with picosecond time resolution. ${ }^{64,65}$ Used in conjunction with simulation, ${ }^{66}$ it has been used to characterize structural disorder and dynamics in several systems, ${ }^{67,68}$ including insulin. ${ }^{69-72}$

While our study does not provide information about the mechanisms through which insulin binds the receptor, it shows that the B-chain $\mathrm{N}$ - and C-termini readily detach. Single-particle cryo-electron microscopy (cryo-EM) structures of the insulinreceptor complex, ${ }^{4,5}$ as well as photo-crosslinking experiments, ${ }^{73,74}$ show that the B-chain C-terminus must detach from the hydrophobic core to bind the receptor, and sequence changes that decrease $\mathrm{e}^{75-77}$ or increase $\mathrm{e}^{78-80}$ the flexibility of this segment result in lower and higher biological activity, respectively. Although the need for the B-chain $\mathrm{N}$-terminus to reorient for insulin to bind the receptor is not apparent from the structures of the complex, ${ }^{4,5}$ substitutions at $\mathrm{Gly}^{\mathrm{B} 8}$ indicate that binding relies on the B8 $\phi$ dihedral angle accessing values characteristic of those we observe in structures with B1-B7 detachment, ${ }^{52,81,82}$ suggesting the functional importance of this element of disorder as well.

On the other hand, cluster 3 is consistent with NMR structures of wild-type human insulin under amyloidogenic conditions $\left(60^{\circ} \mathrm{C}, \mathrm{pH} 2.4\right),{ }^{83}$ which exhibit disorder in the form of AN-helix melting and B1-B7 detachment. The NMR structures are relatively well-structured at the B-chain Cterminus, though a number of them have B21 $\phi$ dihedral angle values that are consistent with those observed for cluster 3 (Figure S4). Substitutions at $\mathrm{Ile}^{\mathrm{A} 2}, \mathrm{Val}^{\mathrm{A} 3}$, and $\mathrm{Thr}^{\mathrm{A} 8}$ that are expected to favor helix formation reduce thermodynamic stability but increase resistance to fibrillation. Given that it is thought that $\mathrm{A} 2$ and $\mathrm{A} 3$ are conserved because they make receptor contacts, ${ }^{83}$ we speculate that the amount of disorder we observe in our ensemble reflects an evolutionary trade-off between the promotion of receptor binding and fibrillation.

The structures that we observe can serve as starting points for the rational design of insulin analogues. The recent introduction of a thermostable active monomeric single-chain insulin analogue in which the B-chain C-terminus is linked to the Achain $\mathrm{N}$-terminus via a six-residue peptide ${ }^{84}$ shows that there is wide scope for new therapeutics. NMR structures of this complex suggest that a linker of sufficient length is needed to enable conformational change upon receptor binding, pointing to the importance of considering the accessible conformational space in the design. The interplay of B1-B7 with B9-B14 and the heterogeneity at $\mathrm{B} 19-\mathrm{B} 23$, which have received less attention than the $\mathrm{AN}$-helix and B-chain C-terminus, may be worth further consideration for design. The broader question of how to think of intrinsic disorder in molecular association remains open.

\section{ASSOCIATED CONTENT}

\section{Supporting Information}

The Supporting Information is available free of charge at https://pubs.acs.org/doi/10.1021/acs.biochem.1c00583.

Additional methods and Supporting Information Figures ( $\mathrm{S} 1-\mathrm{S} 12$, as referenced in the text), as well as coordinate files for 10 sample structures for each cluster (0-9) and 10 sample structures for each microstate in the MSM (PDF)

\section{AUTHOR INFORMATION}

\section{Corresponding Authors}

Andrei Tokmakoff - Department of Chemistry, The University of Chicago, Chicago, Illinois 60637, United States; James Franck Institute and Institute for Biophysical Dynamics, The University of Chicago, Chicago, Illinois 60637, United States; 다이.org/0000-0002-2434-8744; Email: tokmakoff@ uchicago.edu

Aaron R. Dinner - Department of Chemistry, The University of Chicago, Chicago, Illinois 60637, United States; James Franck Institute and Institute for Biophysical Dynamics, The University of Chicago, Chicago, Illinois 60637, United States; ○ orcid.org/0000-0001-8328-6427; Email: dinner@ uchicago.edu 


\section{Authors}

Luis Busto-Moner - Department of Chemistry, The University of Chicago, Chicago, Illinois 60637, United States

Chi-Jui Feng - Department of Chemistry, The University of Chicago, Chicago, Illinois 60637, United States; (1) orcid.org/ 0000-0002-4006-9489

Adam Antoszewski - Department of Chemistry, The University of Chicago, Chicago, Illinois 60637, United States; (1) orcid.org/0000-0003-0097-5257

Complete contact information is available at: https://pubs.acs.org/10.1021/acs.biochem.1c00583

\section{Notes}

The authors declare no competing financial interest.

\section{ACKNOWLEDGMENTS}

The authors thank Jonathan Weare, Robert Webber, and Chatipat Lorpaiboon for helpful discussions on IVAC and MSM construction and Michael Weiss and Markus Meuwly for helpful discussions regarding protection factors. L.B.-M. thanks Luis Jordá Bordoy for his help in the use of GROMACS functionalities. This work was supported by the National Institute of General Medical Sciences of the National Institutes of Health (Award No. R35 GM136381 to A.R.D. and Award No. R01 GM118774 to A.T.). Computations were performed on the GM4 cluster supported by the University of Chicago Research Computing Center and funded by the National Science Foundation Major Research Instrumentation Award DMR1828629 .

\section{REFERENCES}

(1) Zaykov, A. N.; Mayer, J. P.; DiMarchi, R. D. Pursuit of a Perfect Insulin. Nat. Rev. Drug Discovery 2016, 15, 425-439.

(2) Petersen, M. C.; Shulman, G. I. Mechanisms of Insulin Action and Insulin Resistance. Physiological Reviews 2018, 98, 2133-2223.

(3) DeFelippis, M. R.; Chance, R. E.; Frank, B. H. Insulin SelfAssociation and the Relationship to Pharmacokinetics and Pharmacodynamics. Crit. Rev. Ther. Drug Carrier Syst. 2001, 18, 201-264.

(4) Scapin, G.; et al. Structure of the Insulin Receptor-Insulin Complex by Single-Particle Cryo-EM Analysis. Nature 2018, 556, 122125.

(5) Weis, F.; et al. The Signalling Conformation of the Insulin Receptor Ectodomain. Nat. Commun. 2018, 9, No. 4420.

(6) Brange, J.; Andersen, L.; Laursen, E. D.; Meyn, G.; Rasmussen, E. Toward Understanding Insulin Fibrillation. J. Pharm. Sci. A 1997, 86, 517-525.

(7) Nielsen, L.; Khurana, R.; Coats, A.; Frokjaer, S.; Brange, J.; Vyas, S.; Uversky, V. N.; Fink, A. L. Effect of Environmental Factors on the Kinetics of Insulin Fibril Formation: Elucidation of the Molecular Mechanism. Biochemistry 2001, 40, 6036-6046.

(8) Raskin, P.; Holcombe, J. H.; Tamborlane, W. V.; Malone, J. I.; Strowig, S.; Ahern, J. A.; Lavent, F. A Comparison of Insulin Lispro and Buffered Regular Human Insulin Administered via Continuous Subcutaneous Insulin Infusion Pump. J. Diabetes Complications 2001, $15,295-300$.

(9) Wolpert, H. A.; Faradji, R. N.; Bonner-Weir, S.; Lipes, M. A. Metabolic Decompensation in Pump Users due to Lispro Insulin Precipitation. BMJ 2002, 324, 1253.

(10) Brange, J.; Langkjoer, L. Insulin Structure and Stability. In Pharmaceutical Biotechnology, 1993; Vol. 5, pp 315-350.

(11) Bocian, W.; Sitkowski, J.; Bednarek, E.; Tarnowska, A.; Kawęcki, R.; Kozerski, L. Structure of Human Insulin Monomer in Water/ Acetonitrile Solution. J. Biomol. NMR 2008, 40, 55-64.

(12) Baker, E. N.; et al. The Structure of 2Zn Pig Insulin Crystals at 1.5 Å Resolution. Philos. Trans. R. Soc., B 1988, 319, 369-456.
(13) Ciszak, E.; Beals, J. M.; Frank, B. H.; Baker, J. C.; Carter, N. D.; Smith, G. Role of C-terminal B-chain Residues in Insulin Assembly: The Structure of Hexameric Lys $^{\mathrm{B} 28}$ Pro $^{\mathrm{B} 29}$-Human Insulin. Structure 1995, 3, 615-622.

(14) Campbell, R. K.; Campbell, L. K.; White, J. R. Insulin Lispro: Its Role in the Treatment of Diabetes Mellitus. Ann. Pharmacother. 1996, $30,1263-1271$.

(15) Hua, Q. X.; Jia, W.; Weiss, M. A. Conformational Dynamics of Insulin. Front. Endocrinol. 2011, 2, 48.

(16) Weiss, M. A.; Hua, Q. X.; Lynch, C. S.; Frank, B. H.; Shoelson, S. E. Heteronuclear 2D NMR Studies of an Engineered Insulin Monomer: Assignment and Characterization of the Receptor-Binding Surface by Selective ${ }^{2} \mathrm{H}$ and ${ }^{13} \mathrm{C}$ Labeling with Application to Protein Design. Biochemistry 1991, 30, 7373-7389.

(17) Hua, Q. X.; Weiss, M. A. Comparative 2D NMR Studies of Human Insulin and Des-pentapeptide insulin: Sequential Resonance Assignment and Implications for Protein Dynamics and Receptor Recognition. Biochemistry 1991, 30, 5505-5515.

(18) Bonomi, M.; Heller, G. T.; Camilloni, C.; Vendruscolo, M. Principles of Protein Structural Ensemble Determination. Curr. Opin. Struct. Biol. 2017, 42, 106-116.

(19) Henzler-Wildman, K.; Kern, D. Dynamic personalities of proteins. Nature 2007, 450, 964-972.

(20) Baiz, C. R.; Reppert, M.; Tokmakoff, A. In Ultrafast Infrared Vibrational Spectroscopy, Fayer, M. D., Ed.; CRC Press: New York, 2013; pp 361-404.

(21) Dyson, H. J.; Wright, P. E. NMR Illuminates Intrinsic Disorder. Curr. Opin. Struct. Biol. 2021, 70, 44-52.

(22) Schuler, B.; Hofmann, H. Single-Molecule Spectroscopy of Protein Folding Dynamics-Expanding Scope and Timescales. Curr. Opin. Struct. Biol. 2013, 23, 36-47.

(23) Kuzmenkina, E. V.; Heyes, C. D.; Nienhaus, G. U. SingleMolecule Förster Resonance Energy Transfer Study of Protein Dynamics under Denaturing Conditions. Proc. Natl. Acad. Sci. 2005, $102,15471-15476$

(24) Gambin, Y.; Deniz, A. A. Multicolor Single-Molecule FRET to Explore Protein Folding and Binding. Mol. BioSyst. 2010, 6, 15401547.

(25) Zoete, V.; Meuwly, M.; Karplus, M. A Comparison of the Dynamic Behavior of Monomeric and Dimeric Insulin Shows Structural Rearrangements in the Active Monomer. J. Mol. Biol. 2004, 342, 913-929.

(26) Bagchi, K.; Roy, S. Sensitivity of Water Dynamics to Biologically Significant Surfaces of Monomeric Insulin: Role of Topology and Electrostatic Interactions. J. Phys. Chem. B 2014, 118, 3805-3813.

(27) Singh, R.; Bansal, R.; Rathore, A. S.; Goel, G. Equilibrium Ensembles for Insulin Folding from Bias-Exchange Metadynamics. Biophys. J. 2017, 112, 1571-1585.

(28) Hansmann, U. H. E. Parallel Tempering Algorithm for Conformational Studies of Biological Molecules. Chem. Phys. Lett. 1997, 281, 140-150.

(29) Earl, D. J.; Deem, M. W. Parallel Tempering: Theory, Applications, and New Perspectives. Phys. Chem. Chem. Phys. 2005, 7, 3910-3916.

(30) Prinz, J.-H.; Wu, H.; Sarich, M.; Keller, B.; Senne, M.; Held, M.; Chodera, J. D.; Schütte, C.; Noé, F. Markov Models of Molecular Kinetics: Generation and Validation. J. Chem. Phys. 2011, 134, 174105.

(31) Bowman, G. R.; Pande, V. S.; Noé, F. An Introduction to Markov State Models and Their Application to Long Timescale Molecular Simulation. In Advances in Experimental Medicine and Biology, Bowman, G. R.; Pande, V. S.; Noé, F., Eds.; Springer Netherlands: Dordrecht, 2014; Vol. 797, pp 23-44.

(32) Nüske, F.; Keller, B. G.; Pérez-Hernández, G.; Mey, A. S. J. S.; Noé, F. Variational Approach to Molecular Kinetics. J. Chem. Theory Comput. 2014, 10, 1739-1752.

(33) Lorpaiboon, C.; Thiede, E. H.; Webber, R. J.; Weare, J.; Dinner, A. R. Integrated Variational Approach to Conformational Dynamics: A Robust Strategy for Identifying Eigenfunctions of Dynamical Operators. J. Phys. Chem. B 2020, 124, 9354-9364. 
(34) Schütte, C.; Fischer, A.; Huisinga, W.; Deufhard, P. A Direct Approach to Conformational Dynamics Based on Hybrid Monte Carlo. J. Comput. Phys. 1999, 151, 146-168.

(35) MacKerell, A. D.; et al. All-Atom Empirical Potential for Molecular Modeling and Dynamics Studies of Proteins. J. Phys. Chem. B 1998, 102, 3586-3616.

(36) Best, R. B.; Zhu, X.; Shim, J.; Lopes, P. E. M.; Mittal, J.; Feig, M.; MacKerell, A. D. Optimization of the Additive CHARMM All-Atom Protein Force Field Targeting Improved Sampling of the Backbone $\phi, \psi$ and Side-Chain $\chi_{1}$ and $\chi_{2}$ Dihedral Angles. J. Chem. Theory Comput. 2012, 8, 3257-3273.

(37) Huang, J.; Rauscher, S.; Nawrocki, G.; Ran, T.; Feig, M.; De Groot, B. L.; Grubmüller, H.; MacKerell, A. D. CHARMM36m: An Improved Force Field for Folded and Intrinsically Disordered Proteins. Nat. Methods 2017, 14, 71-73.

(38) Abraham, M. J.; Murtola, T.; Schulz, R.; Páll, S.; Smith, J. C.; Hess, B.; Lindah, E. GROMACS: High Performance Molecular Simulations Through Multi-Level Parallelism from Laptops to Supercomputers. SoftwareX 2015, 1-2, 19-25.

(39) Jo, S.; Kim, T.; Iyer, V. G.; Im, W. CHARMM-GUI: A WebBased Graphical User Interface for CHARMM. J. Comput. Chem. 2008, $29,1859-1865$.

(40) Lee, J.; et al. CHARMM-GUI Input Generator for NAMD, GROMACS, AMBER, OpenMM, and CHARMM/OpenMM Simulations Using the CHARMM36 Additive Force Field. J. Chem. Theory Comput. 2016, 12, 405-413.

(41) Humphrey, W.; Dalke, A.; Schulten, K. VMD: Visual Molecular Dynamics. J. Mol. Graphics 1996, 14, 33-38.

(42) Goga, N.; Rzepiela, A. J.; De Vries, A. H.; Marrink, S. J.; Berendsen, H. J. Efficient Algorithms for Langevin and DPD Dynamics. J. Chem. Theory Comput. 2012, 8, 3637-3649.

(43) Hess, B.; Bekker, H.; Berendsen, H. J.; Fraaije, J. G. LINCS: A Linear Constraint Solver for Molecular Simulations. J. Comput. Chem. 1997, 18, 1463-1472.

(44) Darden, T.; York, D.; Pedersen, L. Particle Mesh Ewald: An N. $\log (\mathrm{N})$ Method for Ewald Sums in Large Systems. J. Chem. Phys. 1993, 98, 10089-10092.

(45) Jorgensen, W. L.; Chandrasekhar, J.; Madura, J. D.; Impey, R. W.; Klein, M. L. Comparison of Simple Potential Functions for Simulating Liquid Water. J. Chem. Phys. 1983, 79, 926-935.

(46) Søndergaard, C. R.; Olsson, M. H. M.; Rostkowski, M.; Jensen, J. $\mathrm{H}$. Improved Treatment of Ligands and Coupling Effects in Empirical Calculation and Rationalization of $\mathrm{p} K_{\mathrm{a}}$ Values. J. Chem. Theory Comput. 2011, 7, 2284-2295.

(47) Olsson, M. H. M.; Søndergaard, C. R.; Rostkowski, M.; Jensen, J. H. PROPKA3: Consistent Treatment of Internal and Surface Residues in Empirical $\mathrm{pK}_{\mathrm{a}}$ Predictions. J. Chem. Theory Comput. 2011, 7, 525537.

(48) Parrinello, M.; Rahman, A. Polymorphic Transitions in Single Crystals: A New Molecular Dynamics Method. J. Appl. Phys. 1981, 52, 7182-7190.

(49) Patriksson, A.; van der Spoel, D. A temperature predictor for parallel tempering simulations. Phys. Chem. Chem. Phys. 2008, 10, 2073-2077.

(50) Scherer, M. K.; Trendelkamp-Schroer, B.; Paul, F.; PérezHernández, G.; Hoffmann, M.; Plattner, N.; Wehmeyer, C.; Prinz, J.H.; Noé, F. PyEMMA 2: A Software Package for Estimation, Validation, and Analysis of Markov Models. J. Chem. Theory Comput. 2015, 11, 5525-5542.

(51) Park, I.-H.; Venable, J. D.; Steckler, C.; Cellitti, S. E.; Lesley, S. A.; Spraggon, G.; Brock, A. Estimation of Hydrogen-Exchange Protection Factors from MD Simulation Based on Amide Hydrogen Bonding Analysis. J. Chem. Inf. Model. 2015, 55, 1914-1925.

(52) Hua, Q.-X.; Nakagawa, S.; Hu, S.-Q.; Jia, W.; Wang, S.; Weiss, M. A. Toward the Active Conformation of Insulin: Stereospecific Modulation of a Structural Switch in the B Chain. J. Biol. Chem. 2006, 281, 24900-24909.

(53) Kubelka, J.; Hofrichter, J.; Eaton, W. A. The Protein Folding 'Speed Limit'. Curr. Opin. Struct. Biol. 2004, 14, 76-88.
(54) Bastian, M.; Heymann, S.; Jacomy, M. Gephi: An Open Source Software for Exploring and Manipulating Networks, Third International AAAI Conference on Weblogs and Social Media, 2009; pp 361-362.

(55) Hvidt, A.; Linderstrøm-Lang, K. Exchange of Hydrogen Atoms in Insulin with Deuterium Atoms in Aqueous Solutions. BBA - Biochim. Biophys. Acta 1954, 14, 574-575.

(56) Hvidt, A.; Nielsen, S. O. Hydrogen Exchange in Proteins. In Advances in Protein Chemistry, Anfinsen, C.; Anson, M.; Edsall, J. T.; Richards, F. M., Eds.; Academic Press, 1966; Vol. 21, pp 287-386.

(57) Englander, S. W.; Mayne, L.; Bai, Y.; Sosnick, T. R. Hydrogen Exchange: The Modern Legacy of Linderstrøm-Lang. Protein Sci. 1997, 6, 1101-1109.

(58) Englander, S. W.; Kallenbach, N. R. Hydrogen Exchange and Structural Dynamics of Proteins and Nucleic Acids. Q. Rev. Biophys. 1983, 16, 521-655.

(59) Mohammadiarani, H.; Shaw, V. S.; Neubig, R. R.; Vashisth, H. Interpreting Hydrogen-Deuterium Exchange Events in Proteins Using Atomistic Simulations: Case Studies on Regulators of G-Protein Signaling Proteins. J. Phys. Chem. B 2018, 122, 9314-9323.

(60) Bryant, C.; Spencer, D. B.; Miller, A.; Bakaysa, D. L.; McCune, K. S.; Maple, S. R.; Pekar, A. H.; Brems, D. N. Acid Stabilization of Insulin. Biochemistry 1993, 32, 8075-8082.

(61) Smith, M. D.; Rao, J. S.; Segelken, E.; Cruz, L. Force-Field Induced Bias in the Structure of $\mathrm{A} \beta_{21-30}$ : A Comparison of OPLS, AMBER, CHARMM, and GROMOS Force Fields. J. Chem. Inf. Model. 2015, 55, 2587-2595.

(62) Rauscher, S.; Gapsys, V.; Gajda, M. J.; Zweckstetter, M.; De Groot, B. L.; Grubmüller, H. Structural Ensembles of Intrinsically Disordered Proteins Depend Strongly on Force Field: A Comparison to Experiment. J. Chem. Theory Comput. 2015, 11, 5513-5524.

(63) Eliezer, D. Biophysical Characterization of Intrinsically Disordered Proteins. Curr. Opin. Struct. Biol. 2009, 19, 23-30.

(64) Dhayalan, B.; Fitzpatrick, A.; Mandal, K.; Whittaker, J.; Weiss, M. A.; Tokmakoff, A.; Kent, S. B. H. Efficient Total Chemical Synthesis of ${ }^{13} \mathrm{C}={ }^{18} \mathrm{O}$ Isotopomers of Human Insulin for Isotope-Edited FTIR. ChemBioChem 2016, 17, 415-420.

(65) Smith, A. W.; Lessing, J.; Ganim, Z.; Peng, C. S.; Tokmakoff, A.; Roy, S.; Jansen, T. L. C.; Knoester, J. Melting of a $\beta$-Hairpin Peptide Using Isotope-Edited 2D IR Spectroscopy and Simulations. J. Phys. Chem. B 2010, 114, 10913-10924.

(66) Reppert, M.; Tokmakoff, A. Computational Amide I 2D IR Spectroscopy as a Probe of Protein Structure and Dynamics. Annu. Rev. Phys. Chem. 2016, 67, 359-386.

(67) Mukherjee, P.; Kass, I.; Arkin, I. T.; Zanni, M. T. Structural Disorder of the CD3 $\zeta$ Transmembrane Domain Studied with 2D IR Spectroscopy and Molecular Dynamics Simulations. J. Phys. Chem. B 2006, 110, 24740-24749.

(68) Reppert, M.; Roy, A. R.; Tempkin, J. O. B.; Dinner, A. R.; Tokmakoff, A. Refining Disordered Peptide Ensembles with Computational Amide I Spectroscopy: Application to Elastin-Like Peptides. J. Phys. Chem. B 2016, 120, 11395-11404.

(69) Ganim, Z.; Jones, K. C.; Tokmakoff, A. Insulin Dimer Dissociation and Unfolding Revealed by Amide I Two-Dimensional Infrared Spectroscopy. Phys. Chem. Chem. Phys. 2010, 12, 3579-3588.

(70) Salehi, S. M.; Koner, D.; Meuwly, M. Dynamics and Infrared Spectrocopy of Monomeric and Dimeric Wild Type and Mutant Insulin. J. Phys. Chem. B 2020, 124, 11882-11894.

(71) Antoszewski, A.; Feng, C. J.; Vani, B. P.; Thiede, E. H.; Hong, L.; Weare, J.; Tokmakoff, A.; Dinner, A. R. Insulin Dissociates by Diverse Mechanisms of Coupled Unfolding and Unbinding. J. Phys. Chem. B 2020, 124, 5571-5587.

(72) Feng, C.-J.; Sinitskiy, A.; Pande, V.; Tokmakoff, A. Computational IR Spectroscopy of Insulin Dimer Structure and Conformational Heterogeneity. J. Phys. Chem. B 2021, 125, 4620-4633.

(73) Menting, J. G.; et al. How Insulin Engages its Primary Binding Site on the Insulin Receptor. Nature 2013, 493, 241-245.

(74) Xu, Y.; Kong, G. K.-W.; Menting, J. G.; Margetts, M. B.; Delaine, C. A.; Jenkin, L. M.; Kiselyov, V. V.; De Meyts, P.; Forbes, B. E.; 
Lawrence, M. C. How Ligand Binds to the Type 1 Insulin-like Growth

Factor Receptor. Nat. Commun. 2018, 9, No. 821.

(75) Markussen, J.; Jørgensen, K. H.; Sørensen, A. R.; Thim, L. Single Chain Des-(B30) Insulin. Int. J. Peptide Protein Res. 1985, 26, 70-77.

(76) Derewenda, U.; Derewenda, Z.; Dodson, E. J.; Dodson, G. G.; Bing, X.; Markussen, J. X-ray Analysis of the Single Chain B29-A1 Peptide-linked Insulin Molecule: A Completely Inactive Analogue. J. Mol. Biol. 1991, 220, 425-433.

(77) Hua, Q. X.; Shoelson, S. E.; Kochoyan, M.; Weiss, M. A. Receptor Binding Redefined by a Structural Switch in a Mutant Human Insulin. Nature 1991, 354, 238-241.

(78) Hua, Q. X.; Xu, B.; Huang, K.; Hu, S. Q.; Nakagawa, S.; Jia, W.; Wang, S.; Whittaker, J.; Katsoyannis, P. G.; Weiss, M. A. Enhancing the Activity of a Protein by Stereospecific Unfolding: Conformational Life cycle of Insulin and its Evolutionary Origins. J. Biol. Chem. 2009, 284, 14586-14596.

(79) Žáková, L.; Kletvíková, E.; Veverka, V.; Lepšík, M.; Watson, C. J.; Turkenburg, J. P.; Jiráček, J.; Brzozowski, A. M. Structural Integrity of the B24 Site in Human Insulin is Important for Hormone Functionality. J. Biol. Chem. 2013, 288, 10230-10240.

(80) Keller, D.; Clausen, R.; Josefsen, K.; Led, J. J. Flexibility and Bioactivity of Insulin: an NMR Investigation of the Solution Structure and Folding of an Unusually Flexible Human Insulin Mutant with Increased Biological Activity. Biochemistry 2001, 40, 10732-10740.

(81) Nakagawa, S. H.; Zhao, M.; Hua, Q.-x.; Hu, S.-Q.; Wan, Z.-1.; Jia, W.; Weiss, M. A. Chiral Mutagenesis of Insulin. Foldability and Function Are Inversely Regulated by a Stereospecific Switch in the B Chain. Biochemistry 2005, 44, 4984-4999.

(82) Guo, Z.-Y.; Zhang, Z.; Jia, X.-Y.; Tang, Y.-H.; Feng, Y.-M. Mutational Analysis of the Absolutely Conserved B8Gly: Consequence on Foldability and Activity of Insulin. Acta Biochim. Biophys. Sin. 2005, $37,673-679$.

(83) Hua, Q.-X.; Weiss, M. A. Mechanism of Insulin Fibrillation: The Structure of Insulin under Amyloidogenic Conditions Resembles a Protein-Folding Intermediate. J. Biol. Chem. 2004, 279, 21449-21460.

(84) Glidden, M. D.; et al. Solution Structure of an Ultra-Stable Single-Chain Insulin Analog Connects Protein Dynamics to a Novel Mechanism of Receptor Binding. J. Biol. Chem. 2018, 293, 69-88. 